\title{
LA MEDICINA DEL TRABAJO EN LOS MEDICAL SUBJECT HEADING TERMS (MESH) Y LOS DESCRIPTORES DE CIENCIAS DE LA SALUD (DECS)
}

\author{
JAVIER SANZ-VALERO*,**, CARLOS ROJO-ALONSO*** \\ (*) Departamento de Enfermería Comunitária, Medicina Preventiva y Salud Pública e \\ Historia de la Ciencia. Universidad de Alicante. Alicante. España. \\ (**) Departamento de Salud Pública, Historia de la Ciencia y Ginecología. \\ Universidad Miguel Hernández. Elche. España. \\ $(* * *)$ Departamento de Medicina del Trabajo. \\ Consellería de Educación - Generalitat Valenciana. Alicante. España
}

\section{Sr. Director:}

La recuperación de la literatura científica, con un alto grado de pertinencia y sensibilidad, se ha convertido en una labor más de cualquier investigador en la esfera de las ciencias de la salud. Estar al tanto de los referentes científicos es situación primordial a la hora de resolver cualquier problema que se plantee en el curso de la actividad profesional. Para ello, como es conocido, la utilización de descriptores es fundamental a la hora de indizar un artículo científico para su archivo en la bases de datos bibliográficas nacionales (IBECS, IME) e internacionales (MEDLINE, EMBASE, LILACS, SciELO, etc $)^{1}$. Pero mucha mayor importancia adquiere, si cabe, cuando se consultan estas bases para recuperar los documentos.

Desde esta seguridad, hay que destacar que en Enero de 2008 se ha incluido, en el Tesauro de la U.S. National Library of Medicine, un nuevo des-

\section{BIBLIOGRÁFÍA}

1. Veiga de Cabo J. Medicina y Seguridad del Trabajo ha superado los criterios de selección para integrarse en la Red SciELO (Scientific Electronic Library Online). Med Segur Trab. 2007; 3(207):1-4.

2. MeSH Browser [base de datos en Internet]. Bethesda: National Library of Medicine (USA); 1999 [actualizada: 5 oct 2007; acceso 22 feb 2008]. Medical Subject Headings, Main Headings [aproximadamente 5 pantallas]. Disponible en:

http://www.nlm.nih.gov/mesh/2007/MBrowser.html criptor $(\mathrm{MeSH})^{2}$ «Occupational Health Physicians (Médicos del Trabajo): Médicos empleados en una compañía o corporación que no pertenece directamente al cuidado de la salud», rúbrica de la creciente importancia de esta área de la medicina.

Ahora bien, para aumentar la visibilidad de la producción científica es necesario conocer y utilizar adecuadamente todos los descriptores relacionados con la Medicina del Trabajo. Así, desde este breve escrito queremos alentar el estudio de los descriptores aplicables a la Medicina del Trabajo, tanto MeSH como $\mathrm{DeSC}^{3}$, como elementos indispensables en la actualización de conocimientos en la práctica profesional o como herramientas útiles en la revisión del conocimiento (artículos de revisión). Al tiempo, para investigar y conocer, desde el punto de vista de la documentación científica, la aportación de la Medicina del Trabajo dentro del conjunto de las ciencias de la salud.
3. Descriptores en Ciencias de la Salud (DeCS) [Base de datos en Internet]. Sao Paulo: Biblioteca Virtual en Salud BIREME/OPS; 1999. [acceso 23 feb 2008]. Disponible en:

http://decs.bvs.br/E/homepagee.htm 